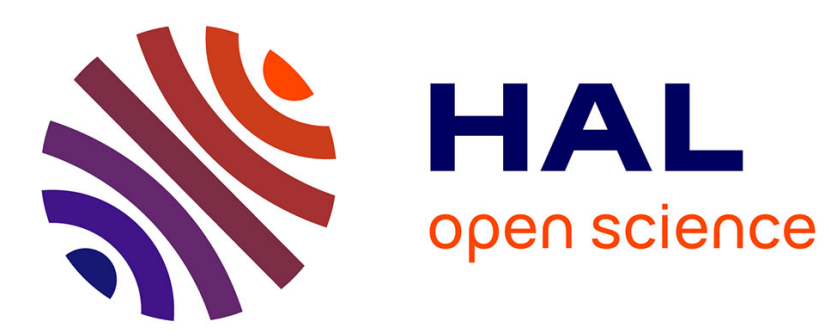

\title{
Global mode of a sphere turbulent wake controlled by a small sphere
}

G Vilaplana, M Grandemange, M Gohlke, Olivier Cadot

\section{To cite this version:}

G Vilaplana, M Grandemange, M Gohlke, Olivier Cadot. Global mode of a sphere turbulent wake controlled by a small sphere. Journal of Fluids and Structures, 2013, 10.1016/j.jfluidstructs.2013.02.022 . hal-01228159

\section{HAL Id: hal-01228159 \\ https://hal-ensta-paris.archives-ouvertes.fr/hal-01228159}

Submitted on 12 Nov 2015

HAL is a multi-disciplinary open access archive for the deposit and dissemination of scientific research documents, whether they are published or not. The documents may come from teaching and research institutions in France or abroad, or from public or private research centers.
L'archive ouverte pluridisciplinaire HAL, est destinée au dépôt et à la diffusion de documents scientifiques de niveau recherche, publiés ou non, émanant des établissements d'enseignement et de recherche français ou étrangers, des laboratoires publics ou privés. 


\title{
Global mode of a sphere turbulent wake controlled by a small sphere
}

\author{
G. Vilaplana ${ }^{\mathrm{a}}$, M. Grandemange ${ }^{\mathrm{a}, \mathrm{b}}, \mathrm{M}$. Gohlke ${ }^{\mathrm{b}}$, O. Cadot $^{\mathrm{a}, *}$ \\ ${ }^{a}$ Unité de Mécanique, Ecole Nationale Supérieure de Techniques Avancées ParisTech, \\ Chemin de la Hunière, 91761 Palaiseau Cedex, France. \\ ${ }^{b}$ Department of Research and Innovation, PSA Peugeot-Citroën, 2 route de Gisy, 78943 \\ Vélizy-Villacoublay, France.
}

\begin{abstract}
A small control sphere is displaced in a cross flow plane downstream of a main sphere of diameter $D$ in a uniform flow at $R e=33000$. The wake is studied by means of 4 fixed hot wire probes. It is shown that without the control sphere (reference case), vortex loops are shed from only one side of the sphere at a Strouhal number $\mathrm{St}=0.19$. This reference wake structure has a planar symmetry defining an azimuthal phase $\theta_{W}$ that is observed to be random in time. The secondary smaller sphere is treated as a local disturbance of the reference wake. It is found that the shedding occurs closer to the symmetry axis for the control sphere placed at the center of the wake than for the natural case. When the control sphere is off-centered, a subharmonic at half the natural frequency appears and the azimuthal phase becomes imposed by the control sphere position. A pure subharmonic mode is observed when the control sphere reaches the separated shear, suggesting alternative vortex loops shedding from both sides of the wake.
\end{abstract}

Keywords: Sensitivity, Control, Global mode, Turbulent wake

\section{Introduction}

Recent theoretical efforts have allowed the retrieval of the structural sensitivity of the global mode of two-dimensional wakes in the laminar regime

\footnotetext{
*Corresponding author.

Email address: cadot@ensta.fr (O. Cadot) 
(Hill, 1992; Marquet et al., 2008; Luchini et al., 2009) )obtained experimentally by Strykowski and Sreenivasan (1990) and very recently in the turbulent regime (Meliga et al., 2012) obtained in the experiment of Parezanović and Cadot (2012). For three dimensional cases, the theory applied to axisymmetric flows in the laminar regime (Meliga et al., 2009b) predicts a zone of high sensitivity in the recirculation bubble, particularly around the separatrix. Besides this pioneering work, theoretical prediction for three dimensional turbulent wakes still remains an open issue. On the experimental side, Grandemange et al. (2012) examined the sensitivity of a three dimensional symmetric blunt body in the turbulent regime using small objects in the wake. In this experiment, the presence of the supports that hold the axisymmetric body produce a bi-stable wake, where each state is not axisymmetric and has an $\mathrm{m}=1$ azimuthal periodicity. Depending on their geometry, the small objects select the azimuthal phase or force the axisymmetry of the wake.

The goal of the present experiment is to preserve the axisymmetry of the body as much as possible. We have chosen the academical case of the sphere that is well documented in the literature. The dynamics of the separated flow over spheres undergoes several transitions associated with different wake regimes as the Reynolds number increases (Sakamoto and Haniu, 1990; Szaltys et al., 2012). At very low Reynolds numbers, the flow is steady and axisymmetric. A first bifurcation is reported for $\mathrm{Re} \approx 210$ : a steady azimuthal mode $m=1$ appears. A lift force is generated and a pair of counterrotating vortices develops downstream moving the wake off the streamwise axis so that the flow loses its axisymmetry but keeps a planar symmetry. The plane of symmetry defines the azimuthal phase of the mode. A second bifurcation occurs at a higher Reynolds number $R e \approx 277$ : the wake starts oscillating but preserves the same planar symmetry and azimuthal phase. Becoming turbulent, the preference toward this azimuthal phase vanishes and the flow becomes statistically axisymmetric (Mittal et al., 2002). The mixing layer instabilities in the close wake degenerate into large scale vortex loops developing from the end of the recirculation bubble ; the wake oscillates randomly (Taneda, 1978; Sakamoto and Haniu, 1990) and may have a helical structure highly coherent in space (Pao and Kao, 1977; Berger et al., 1990; Yun et al., 2006; Cannon et al., 1993). This unsteady global mode (dominant mode $m=1$ ) is reported at $0.1<$ St $<0.2$ depending on Reynolds numbers.

The present work explores the global mode modification of the turbulent wake due to the presence of a secondary smaller sphere, treated as a local 

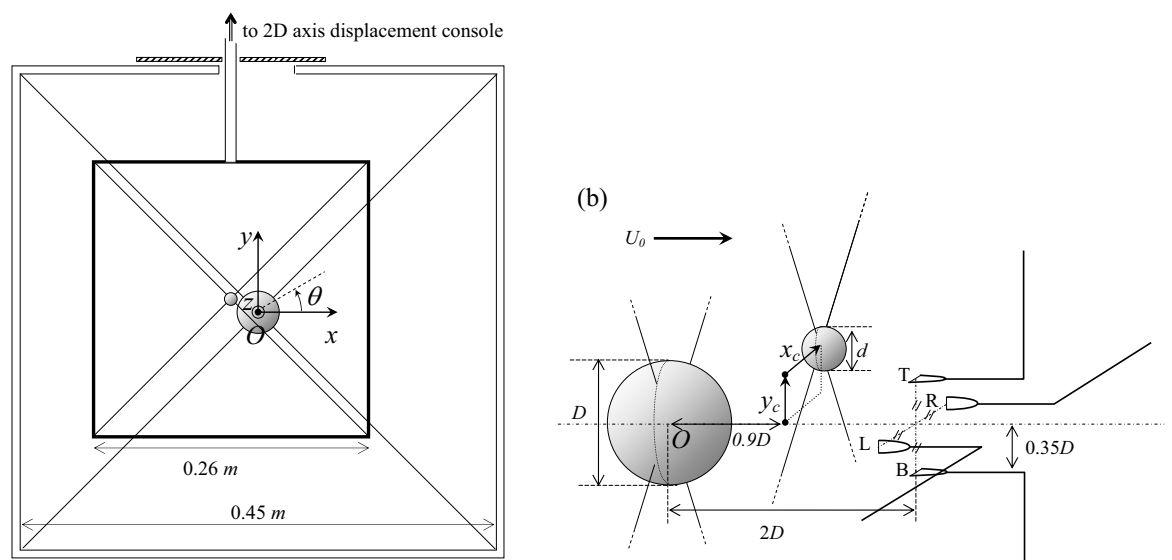

Figure 1: Sketch of the experimental set-up. Backview (a), the flow comes from behind. Four hot wire probes (not on scale) denoted by $\mathrm{L}$ for left, $\mathrm{R}$ for right, $\mathrm{T}$ for top and $\mathrm{B}$ for bottom (directions are related to the backview) are symmetrically positioned 2D downstream from the center of the main sphere (b).

disturbance in its close wake. The impact of the controlled sphere is reported through sensitivity analysis of the global spatial structure and frequency of the wake. To our knowledge, such an approach has not been carried out so far. Wakes interaction have been investigated for identical spheres (Schouveiler et al., 2004; Yoon and Yang, 2007) in the laminar regime around Re $=300$. They lead to different patterns in the vortex shedding and Strouhal numbers, that do not result from a disturbance approach.

The article is organized as follows. First, the experimental set-up and measurements are presented in section 2 . Section 3 analyzes the wake of the main sphere, especially the global mode structure and the topology of the shedding are clarified. Section 4 is devoted to results of the disturbed wake with the small control sphere. Eventually, discussion and concluding remarks are presented in the final section.

\section{Experimental set-up}

The main sphere of diameter $D=40 \mathrm{~mm}$ is placed in a feedback wind tunnel having a uniform velocity of $U_{0}=12.5 \mathrm{~m} / \mathrm{s}$ with a free stream turbulence level of $0.5 \%$. The diameter $D$ and inlet velocity $U_{0}$ are used to build non-dimensional values marked with an asterisk. The Reynolds number is 
$\operatorname{Re}=U_{0} D / \nu=33000$ for the main sphere. The cross section of the wind tunnel is a square of dimensions $0.45 \mathrm{~m} \times 0.45 \mathrm{~m}$ and the sphere is attached from its center to each corner of the test section by four metal wires of diameter $0.5 \mathrm{~mm}$ as depicted in Fig.1. A system of tension at the edge of the frame allows to stretch the wires. The tension is adjusted in order to avoid any observable or hearable vibrations of the wires and the sphere. This technique of fixation is commonly used for studies of three dimensional bluff body wakes, see for instance Cannon et al. (1993); Howe et al. (2001); Jang and Lee (2008); Sakamoto and Haniu (1995).

The control sphere has a diameter of $d=12 \mathrm{~mm}=0.3 D$. The surface ratio between the main sphere and the control sphere is sufficiently small, $(d / D)^{2}=0.09$ to consider the control sphere as a disturbance for the main sphere wake. The control sphere is also attached by four $0.5 \mathrm{~mm}$ metal wires from its center to the corners of a squared moving frame of dimensions $0.26 \mathrm{~m} \times 0.26 \mathrm{~m}$. They are sufficiently stretched to avoid any vibrations. The frame is made by four metal profiles with a cross section of $3 \mathrm{~mm} \times 15 \mathrm{~mm}$ with smooth edges. The upper part of the frame is fixed to a $10 \mathrm{~mm}$ rod driven by a two-axis displacement console standing on the top floor of the test section area. The passage is made air proof by adding heavy sliding sheets between the open part of the roof and the rod. Let $x_{c}, y_{c}, z_{c}$ be the coordinates of the center of the control sphere whose origin is taken at the center of the main sphere. The corresponding polar coordinates will be referred to as $r_{c}$ and $\theta_{c}$. The control sphere is positioned at a fixed downstream distance $z_{c}=0.9 D$ and displaced by steps of $1 \mathrm{~mm}$ in the area of the $x O y$ plane: $\Delta x_{c} \times \Delta y_{c}=1.25 D \times 1.25 D$.

Four fixed hot wire anemometers measure the velocity in a downstream transversal plane at $z^{*}=2$ (Fig. 1b). The four measurement points denoted by $\mathrm{L}, \mathrm{T}, \mathrm{R}$ and $\mathrm{B}$ are located respectively at $(x=-0.35, y=0),(0,0.35)$, $(-0.35,0)$ and $(0,-0.35)$ in dimensionless quantities. The letters are referring to the left, top, right and bottom part of the main sphere seen from the backview in Fig. 1(a). The hot wires are all oriented in such a way to be sensitive to the velocity component comprised in the azimuthal plane $\theta$ (see Fig. 1a). The four velocity time series are simultaneously recorded using Labview software and a National Instrument acquisition board at a sampling frequency of $1 \mathrm{kHz}$. We denote by $u_{i}(t)$ the measurements obtained with the $i=[L, R, T, B]$ hot wire anemometers and $\delta u_{i}(t)$, the velocity fluctuation about the mean. For specific positions of the control sphere, a flying hot wire probe, sensitive to the $(y O z)$ velocity component is used to explore the 

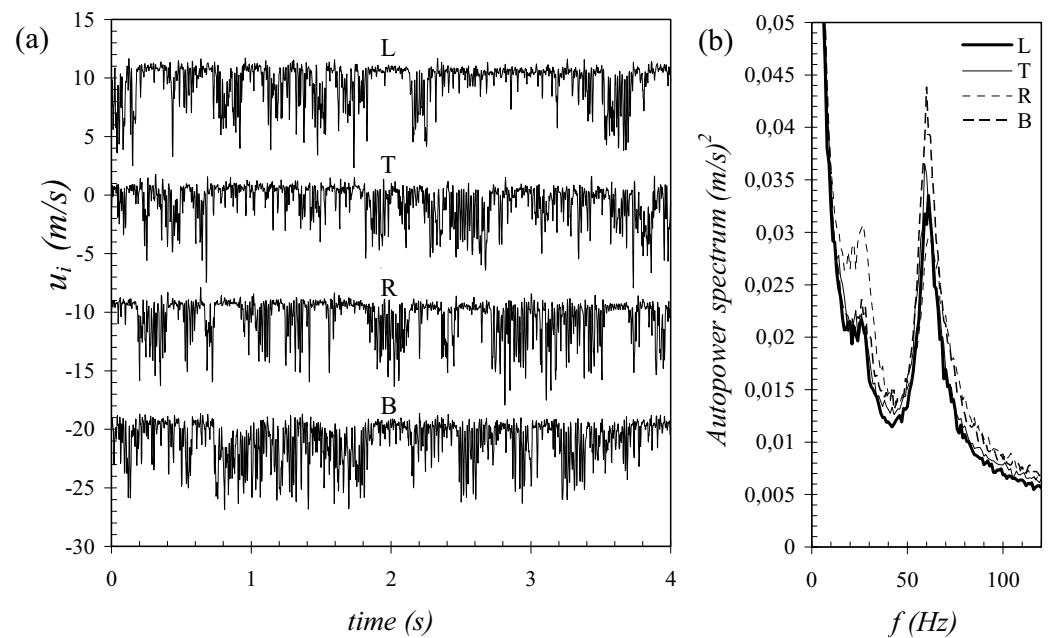

Figure 2: Time series (a) and averaged autopower spectra (b) of the four velocity measurements $u_{i}(t)$ with $i=[L, T, R, B]$ (as depicted in Fig. 1(b)) for the reference wake without control sphere. Except $u_{L}(t)$ in $(\mathrm{a})$, each velocity measurement has been vertically shifted down by a multiple of $10 \mathrm{~m} / \mathrm{s}$.

wake in a transversal plane $z^{*}=2$. In this case the velocity measurement is simply referred to as $u(t)$ and its standard deviation to $u$ rms.

\section{Reference wake}

We first study the case of the main sphere without the control sphere, for which velocities are recorded during $600 \mathrm{~s}$. The time series are very intermittent as shown in Fig. 2(a). One can observe on each signal, time windows of varying durations having lack of fluctuations. The averaged spectra performed over $1 \mathrm{~s}$ duration all present a dominant frequency at $f_{1}=60 \mathrm{~Hz}$. The frequency $f_{1}$, that we will refer to as the mode 1 frequency corresponds to a Strouhal number of 0.19. Another, but less significant characteristic frequency is observed around $f_{2}=27 \mathrm{~Hz}$ which might correspond to a subharmonic of the mode 1 frequency. We computed the coherence functions defined as :

$$
\gamma_{i j}(f)=\frac{\left\langle\tilde{u}_{i}(f) \tilde{u}_{j}^{c c}(f)\right\rangle}{\left\langle\left|\tilde{u}_{i}(f)\right|\right\rangle\left\langle\left|\tilde{u}_{j}(f)\right|\right\rangle},
$$

where $\tilde{u}_{i}(f)$ is the Fourier transform of $u_{i}(t)$, "cc" stands for complex conjugate and the averaging $\langle\ldots\rangle$ is performed over 1s duration. The coherence 

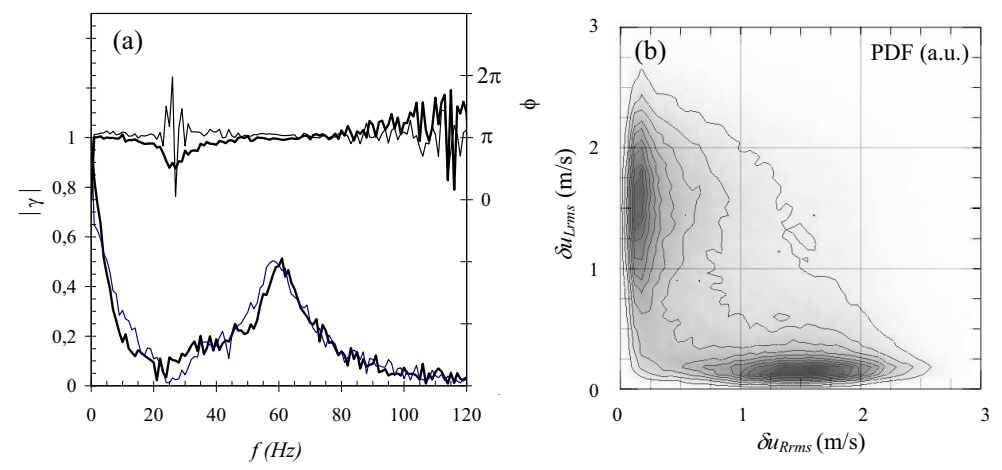

Figure 3: (a): Modulus $|\gamma|$ (bottom) and phase $\phi$ (top) of the horizontal $\gamma_{L R}$ (thick line) and vertical coherence function $\gamma_{T B}$ (thin line). Joint probability density function (b), $\operatorname{PDF}\left(\delta u_{R \mathrm{rms}}, \delta u_{L \mathrm{rms}}\right)$, see text for definitions of the variables $\delta u_{L \mathrm{rms}}$ and $\delta u_{R \mathrm{rms}}$.

is computed between the left and right probes $(i j)=(L R)$ and between the top and bottom probes $(i j)=(T B)$. For simplicity, we will call $\gamma_{L R}$ the horizontal correlation and $\gamma_{T B}$, the vertical correlation. The coherence functions, modulus and phase are displayed in Fig. 3(a). The modulus of the coherence presents a peak around $60 \mathrm{~Hz}$ with a corresponding phase shift of $\pi$ for both the horizontal and vertical coherence functions. The mode 1 can be then viewed as a combination of two equivalent asymmetric oscillations, a vertical one and an horizontal one. However, these oscillations cannot result from an alternative vortex shedding between both side of the wake as commonly observed for the 2D Kármán street. This is demonstrated in the following by considering the time dependant mean fluctuation on each probe over a period $T$ :

$$
\delta u_{i \mathrm{rms}}^{2}(t)=\frac{1}{T} \int_{t}^{t+T}\left[u_{i}(t)-\frac{1}{T} \int_{t}^{t+T} u_{i}(t) d t\right]^{2} d t, \quad i=[L, T, R, B] .
$$

The joint probability distribution $\operatorname{PDF}\left(\delta u_{L \mathrm{rms}}, \delta u_{R \mathrm{rms}}\right)$ calculated over $600 \mathrm{~s}$ is shown in Fig.3(b) for $T=0.025 \mathrm{~s}$ corresponding to 1.5 shedding at $f_{1}=60 \mathrm{~Hz}$. If alternate shedding should occur between the left and right probe, they should record the same averaged velocity fluctuations over 1.5 period and then a single peak on the diagonal of the joint distribution would appear in Fig.3(b). Instead, two symmetric peaks are observed revealing that the shedding is always either located on one side of the wake or on the other side. It confirms the shedding wake structure proposed by Mittal et al. 

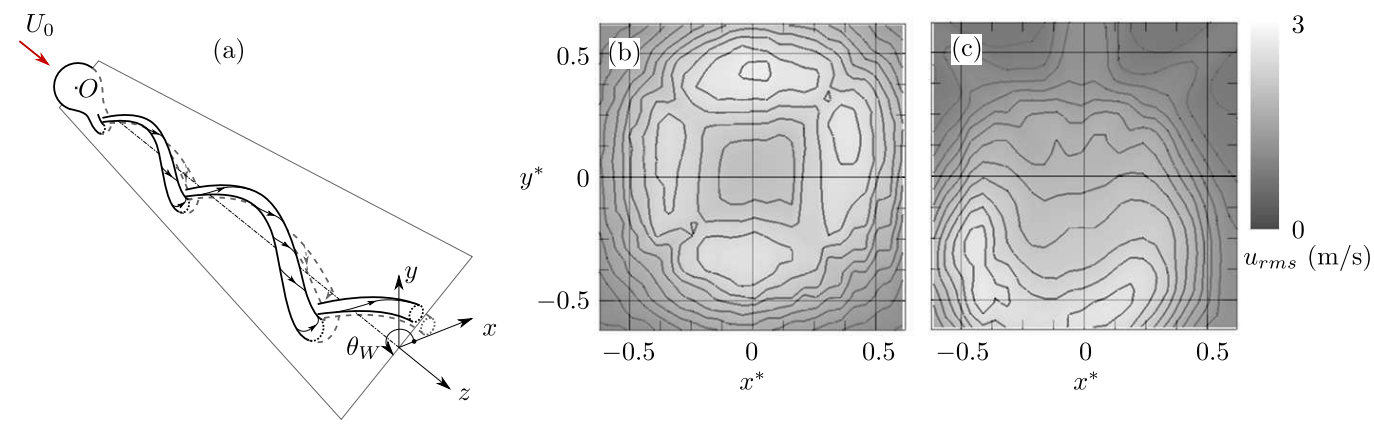

Figure 4: Sketch (a) of the vortex shedding at Strouhal number 0.19. Map of velocity fluctuations $u_{\mathrm{rms}}\left(x^{*}, y^{*}\right)$ in a plane $z^{*}=2$ for (b) natural reference case and (c) with a steady $m=1$ disturbance introduced by a vertical rod placed above the summit of the sphere. The grayscale in (b) and (c) are identical with a contour interval of $0.2 \mathrm{~m} / \mathrm{s}$.

(2002), which consists of vortex loops that are shed from a same side of the wake as sketched in Fig.4(a). This wake structure has a planar symmetry defining the azimuthal phase $\theta_{W}$ of the mode. The random behavior of $\theta_{W}$ in time is responsible for the intermittent behavior of the signals in Fig. 2(a). As also mentioned by Mittal et al. (2002), the random azimuthal phase renders the wake statistically axisymmetric. Using a single probe mounted on the displacement console, we measured the standard deviation of the velocity $u_{\mathrm{rms}}(x, y)$ in the transversal plane $z^{*}=2$. The obtained map in Fig. 4(b) is nearly axisymmetric as expected, although a $m=4$ periodicity due to the support is also observable. We did a test experiment by placing roughly a vertical rod (4 $\mathrm{mm}$ in diameter) from the roof of the test section to the summit of the sphere. The result is shown in Fig. 4(c), the fluctuations concentrate in the opposite region of the rod. The region of large fluctuation reveals the region of the vortex loops shedding and its position defines the azimuthal phase $\theta_{W}$ of the mode. This simple test experiment shows that the random phase can actually be fixed by imposing a strong $m=1$ steady disturbance. It also explains the presence of the $m=4$ periodicity of the reference case in Fig. 4(b) because the four wires used to hold the sphere influence the azimuthal phase as four $m=1$ steady disturbances. The random azimuthal phase cannot be uniformly distributed over all angles $\theta$ and Fig. 4(b) contains four situations as in Fig. 4(c) but shifted of $\pi / 4$. 

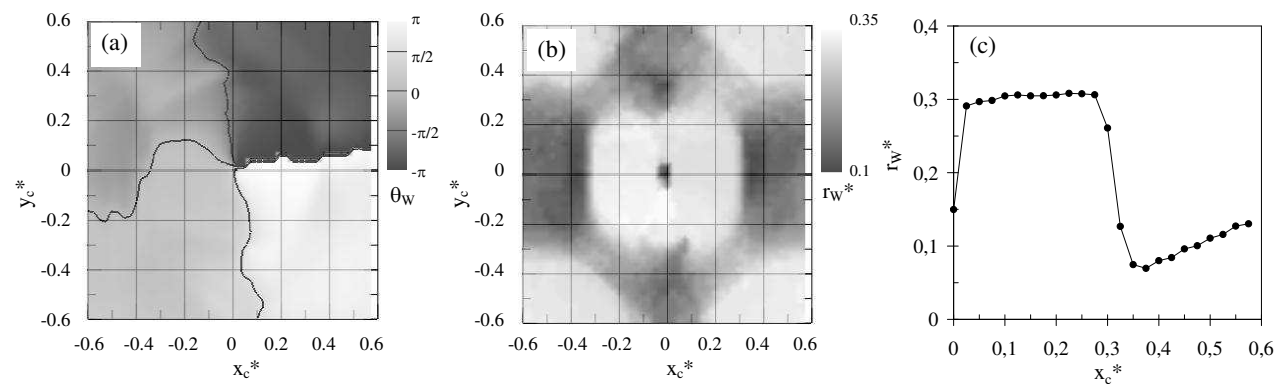

Figure 5: Azimuthal phase of the wake $\theta_{W}$ (a) and radial position of the shedding $r_{W}^{*}$ (b) vs. the position of the control sphere $\left(x_{c}^{*}, y_{c}^{*}\right)$. Radial position of the shedding $r_{W}^{*}(\mathrm{c})$ obtained on the horizontal path $\left(y_{c}^{*}=0\right)$.

\section{Wake with control sphere}

For each position of the control sphere in the transversal plane $z^{*}=0.9$, the four velocity signals captured by the L, T, R, and B probes are recorded during $200 \mathrm{~s}$. At each position, we compute the time dependant fluctuation $\delta u_{i \mathrm{rms}}(t)$ as defined in Eq. 2 with $T=0.025 \mathrm{~s}$. We expect that the barycenter of these four fluctuation signals will locate instantaneously the maximum of the shedding in the transversal plane $z^{*}=2$. The barycenter $\left(x_{F}^{*}(t), y_{F}^{*}(t)\right)$ is defined as :

$$
\begin{aligned}
& x_{F}^{*}(t)=0.35 \frac{\delta u_{R \mathrm{rms}}(t)-\delta u_{L \mathrm{rms}}(t)}{\delta u_{R \mathrm{rms}}(t)+\delta u_{L \mathrm{rms}}(t)}, \\
& y_{F}^{*}(t)=0.35 \frac{\delta u_{T \mathrm{rms}}(t)-\delta u_{B \mathrm{rms}}(t)}{\delta u_{T \mathrm{rms}}(t)+\delta u_{B \mathrm{rms}}(t)} .
\end{aligned}
$$

We measure the azimuthal phase of the shedding $\theta_{W}$ from the mean position of the fluctuation barycenter and its radial position $r_{W}$, as the most probable position of the fluctuation barycenter. For the reference wake, we find $r_{W}^{*}=$ 0.3 .

The control sphere acts as a $m=1$ disturbance except for the position at the center $\left(x_{c}^{*}, y_{c}^{*}\right)=(0,0)$. The azimuthal phase $\theta_{W}$ vs. the position of the control sphere is plotted in Fig. 5(a). We can see that the phase $\theta_{W}$ is related to the sphere polar angle as $\theta_{W} \approx \theta_{c}+\pi$ implying that the shedding always occurs on the opposite side of the control sphere location. The radial position of the shedding $r_{W}^{*}$ is plotted in Fig 5(b). The maps are clearly not axisymmetric but actually contain all the symmetry properties of the experimental set-up (that takes into account holding systems and velocity 

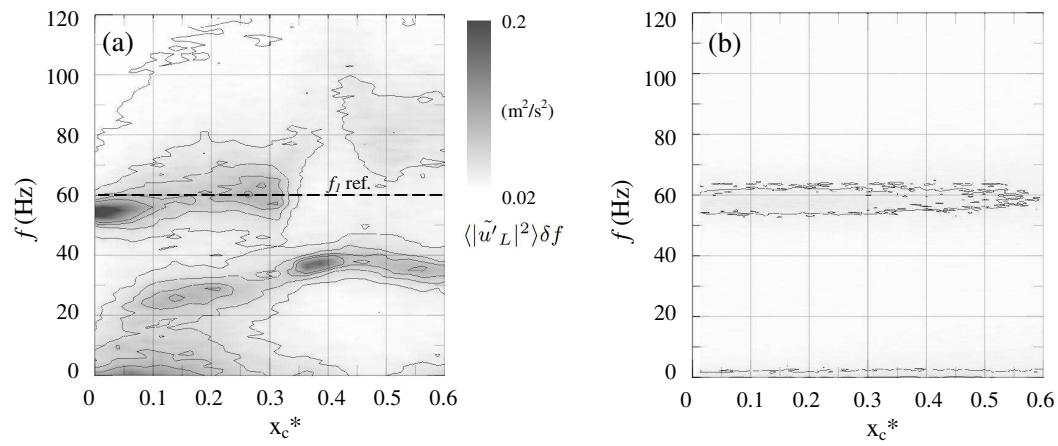

Figure 6: Auto power spectra $\left\langle\left|\tilde{u}_{L}{ }_{L}\left(f, x_{c}^{*}\right)\right|^{2}\right\rangle \delta f$ measured by the L probe vs. the position of the control sphere (a) on the horizontal path $\left(y_{c}^{*}=0\right)$.(b) is the same measurements when the control sphere is removed. It takes into account the obstruction of the moving holding system only (frame and crossed wires see Fig. 1). The 5 contours range from $0.02 \mathrm{~m}^{2} / \mathrm{s}^{-2}$ to $0.1 \mathrm{~m}^{2} / \mathrm{s}^{-2}$ with an interval contour levels of $0.02 \mathrm{~m}^{2} / \mathrm{s}^{-2}$.

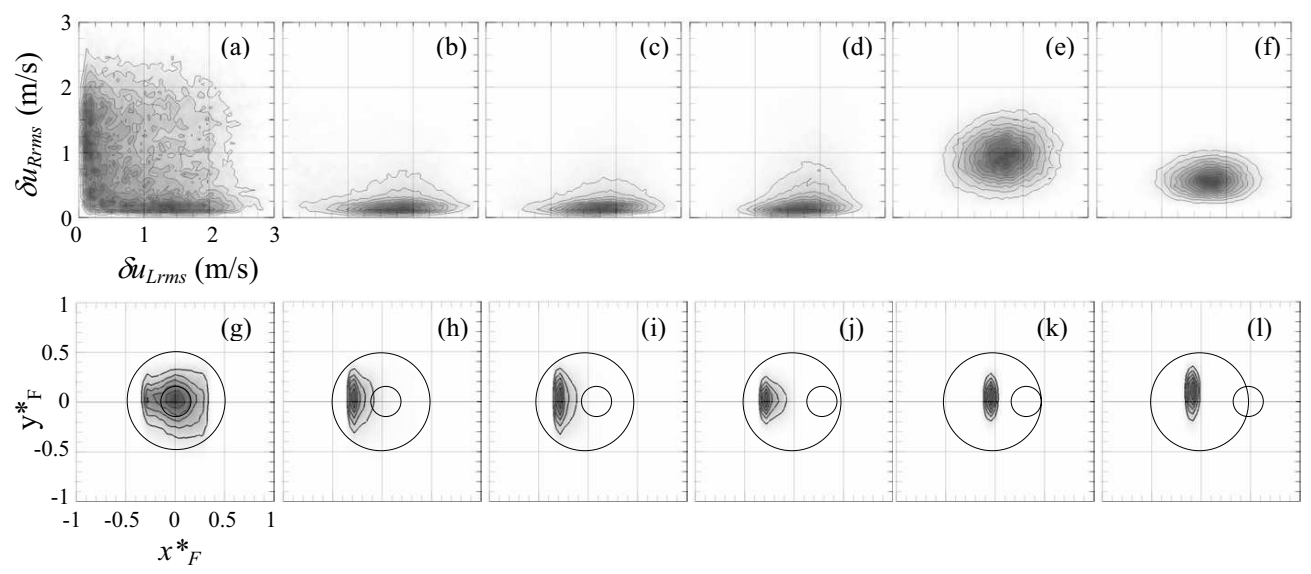

Figure 7: Top raw (a-f) : joint distribution $P D F\left(\delta u_{R \mathrm{rms}}, \delta u_{L \mathrm{rms}}\right)$. Bottom raw (g-l) : joint distribution of the barycenter of the fluctuations $P D F\left(x_{F}^{*}, y_{F}^{*}\right)$. Each column corresponds to a position of the control sphere displaced on an horizontal path $\left(y_{c}^{*}=0\right.$, $\left.x_{c}^{*} \geq 0\right):(\mathrm{a}, \mathrm{g}) x_{c}^{*}=0 ;(\mathrm{b}, \mathrm{h}) x_{c}^{*}=0.07 ;(\mathrm{c}, \mathrm{i}) x_{c}^{*}=0.15 ;(\mathrm{d}, \mathrm{j}) x_{c}^{*}=0.28 ;(\mathrm{e}, \mathrm{k}) x_{c}^{*}=0.35 ;$ $(\mathrm{f}, \mathrm{l}) x_{c}^{*}=0.5$. The empty circles represent the main and the control spheres. 
probes) with four axis of symmetry at $\theta=n \frac{\pi}{4}$ with $n=1 \ldots 4$. On the diagonals, the radial position of the shedding is not very sensitive to the control sphere position. This effect can be explained by the alignment of the wire holding the main sphere with the wire holding the control sphere (see Fig. 1). This special configuration of the wires might create a disturbance as important as the control sphere itself imposing a radial position close to the natural reference case $r_{W}^{*}=0.3$. The largest sensitivity is observed on the horizontal and vertical axis. Figure 5(c) and (d) restrict the sensitivity analysis to the control sphere displaced on an horizontal path $\left(x_{c}^{*}>0, y_{c}^{*}=\right.$ $0)$. Figure 5(c) displays the radial position of the wake $r_{W}^{*}$. Figure $6(\mathrm{a})$ is the auto power spectrum density $\left\langle\left|{\tilde{u^{\prime}}}_{L}\left(f, x_{c}^{*}\right)\right|^{2}\right\rangle$. We only show the spectrum recorded by the probe $\mathrm{L}$ located on the opposite side of the control sphere path (i.e. where the shedding occurs) which actually contains all the spectral information. One can see in Fig. 5(c) that the effect of the control sphere is almost to bring the shedding closer to the symmetry axis compared to the reference case. The closest positions are obtained for the centered control sphere $\left(r_{W}^{*} \approx 0.15\right)$ and off-centered at $r_{c}^{*} \approx 0.37\left(r_{W}^{*} \approx 0.07\right)$. It is also for these two positions that the energy of the fluctuations is more concentrated in the spectra of Fig 6(a) because the shedding is also closer to the measurement points. The two energy concentrations points are around the fundamental frequency $f_{1}=55.5( \pm 1.5) \mathrm{Hz}$ for the centered control sphere and around a lower frequency $f_{2}=38( \pm 1.5) \mathrm{Hz}$ for off-centered control sphere. The modes are pure only for these two cases. Between these two positions, $0.1<r_{c}^{*}<$ 0.35 the radial position $r_{W}$ is similar to that of the reference case and both the fundamental and the low frequency are present. The same measurements have been performed in Fig 6(a) but with a removed control sphere. In that case we can see that the effect of the control sphere supporting system (frame and crossed wires) on the the global frequency is negligible since the natural frequency is retrieved whatever the supporting system position is.

We show in the sequence in Fig. 7(a-f), the joint probability distribution of the time dependant mean fluctuation $\delta u_{i \mathrm{rms}}(t)(\mathrm{Eq} .2$ with $T=0.025 \mathrm{~s}$ ) measured by the right and left probe for different positions of the control sphere. The centered position exhibits the same behavior as for the natural reference wake (Fig. 3) meaning that vortex loops are shed from one side of the wake with a random azimuthal phase. Once the control sphere is slightly shifted (Fig. 7b-d and Fig. 7h-j), the vortex loops are shed from the left side only (opposite location of the control sphere), showing that the azimuthal phase is now fixed to $\theta_{W}=\pi$. The special position corresponding to a 

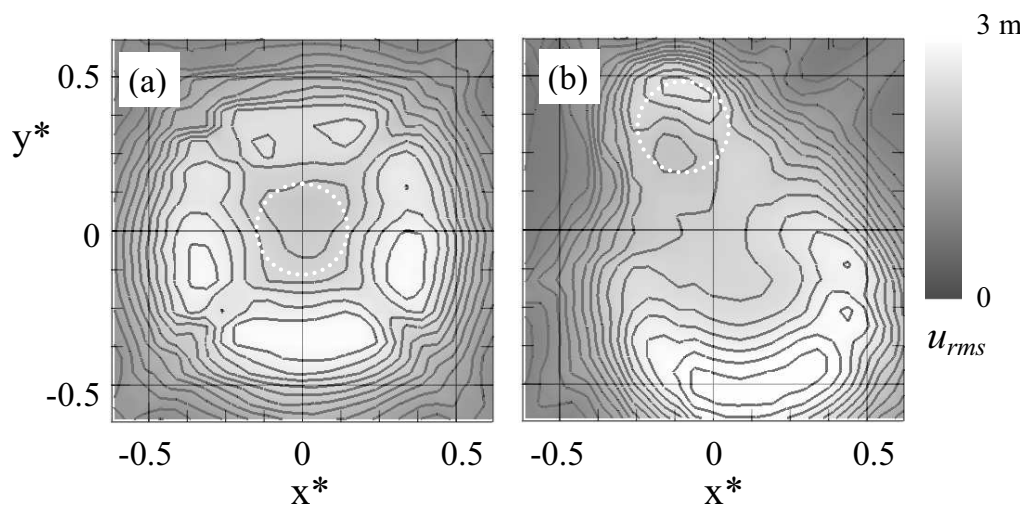

Figure 8: Map of velocity fluctuations $u_{\mathrm{rms}}\left(x^{*}, y^{*}\right)$ in a plane $z^{*}=2$ for (a) centered control sphere and (b) off-centered at $r_{c}^{*}=0.35$. Grayscale and contour interval are identical to Fig 4(b) and (c). The circular white dotted lines in (a) and (b) mark the control sphere.

pure mode at frequency $f_{2}$ is shown in (Fig. 7e,k). The barycenter of the fluctuation (see Fig. 7e) is actually moving back to the center because the fluctuations are no longer recorded by only the left probe, but simultaneously by the left and right probe (see Fig. 7k). This observation associated with the dominance of the subharmonic mode is consistent with an alternate shedding between the side of the control sphere and the opposite side.

Finally, the spatial distribution of the rms fluctuations in the plane $z^{*}=$ 2 measured with the flying probe is shown in Fig. 8 for the two extreme positions $r_{c}^{*}=0$ (Fig. 8a) and $r_{c}^{*}=0.35$ (Fig. 8b). We can actually check that for the centered position, the fluctuations are closer to the wake center compared to the reference case in Fig. 4(b). For the off-centered case we can see two maxima of fluctuations; around the edge of the control sphere and on the opposite side, around the edge of the main sphere.

\section{Discussions and conclusions}

When the control sphere is at the center of the wake of the main sphere, we find similar global properties to that of the reference case, with a pure mode having a frequency close to $\mathrm{St}=0.19$ as described in the sketch of Fig. 4(a) and a random azimuthal phase. The remarkable effect is the rapprochement of the shedding with the center of the wake $\left(r_{W}^{*} \approx 0.15\right.$ with centered control sphere and 0.3 for the reference case). 
(a)

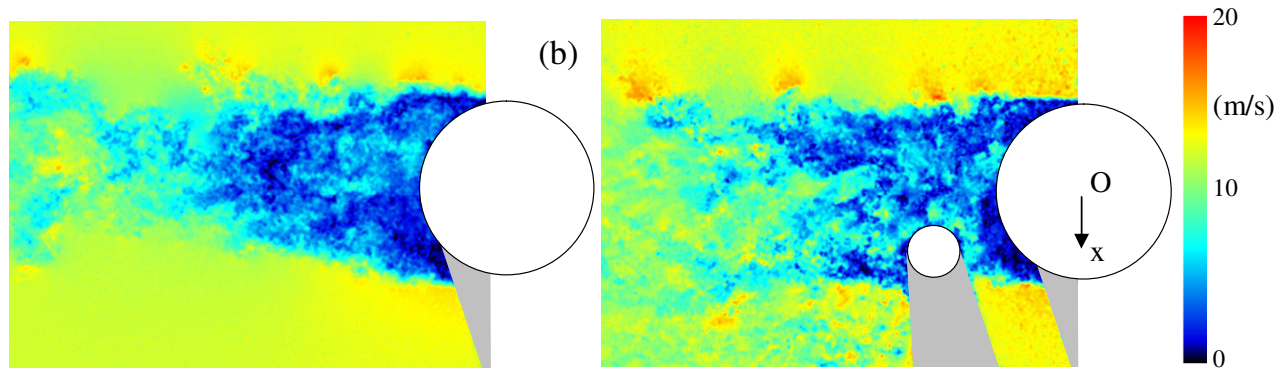

Figure 9: Modulus of the instantaneous velocity measured with Particle Image Velocimetry, (a) without control sphere, (b) control sphere at $x_{c}^{*}=0.35$.

In the cases of the off-centered control sphere, the corresponding disturbance which has an $m=1$ wavenumber sets the turbulent wake at a fixed azimuthal phase. This result generalizes to an axisymmetric situation, the previous observation of Grandemange et al. (2012) for which only two azimuthal phase were observable due to the properties of the natural wake. It was also found that depending on the downstream distance of the perturbation to the body, the shedding could be either on the same side of the steady perturbation or on the opposite side. In the present experiment we only explore one downstream location $z_{c}^{*}=0.9$. For radial positions such that $r_{c}^{*}<0.3$ the shedding occurs on the opposite side of the control sphere location $\theta_{W}=\theta_{c}+\pi$. We do not exclude that for different downstream positions, azimuthal phase of $\theta_{W}=\theta_{c}$ could be observed. In any cases, the fixation of the azimuthal phase by a $m=1$ steady disturbance is a property known for laminar wake that induces the so-called imperfect bifurcation (Meliga et al., 2009a). This major effect avoids the investigation of a complete experimental sensitivity analysis of the global mode using a steady disturbance as in Parezanović and Cadot (2012), since it seems impossible to place the disturbance anywhere else than in the plane of planar symmetry of the mode. The control sphere also modifies the spectral content of the fluctuations and a clear transition between two modes having a factor two in frequency is observed. At the centered position, the global frequency is close to that of the natural wake. A subharmonic, at half the global frequency appears as soon as the control sphere is off-centered. Once the radial position of the sphere reaches $r_{c}^{*}=0.3$ only the subharmonic mode is observed (Fig. $5 \mathrm{~d}$ ). We can explain the factor $1 / 2$ in the frequency jump by the appearance of a new shedding pattern, for which vortex loops are not shed from mostly one side of the 
wake as for the reference wake, but alternatively from the edge of the control sphere and the edge of the main sphere. These two positions can be repaired by the two maxima of velocity fluctuations in Fig.8(b). To illustrate this interpretation, we show instantaneous velocity fields in Fig. 9 measured with a particle image velocimetry. For the reference wake in Fig. 9(a), the vortex loops are observable at the top of the field by the periodic over-velocities in red color. In the second field in Fig. 9(a), over-velocities are distributed alternatively at the top and at the bottom of the wake.

It is noteworthy to mention that the subharmonic frequency is also observable in Fig. 2(b) for the reference wake but at a low amplitude. The alternate vortex loops shedding mode should also exists for the natural wake, meaning that the wake might switch during small duration from the single sided vortex loops shedding (mode 1) to the alternative vortex loops shedding (mode 2 ). The control sphere plays a role in the selection of the dominant mode.

We also observed slight but significant variations of the frequencies of the modes in Fig. 5(b). Whatever the mode, the frequency is increased when the control sphere approaches the inner part of the separated layers. This seems to be consistent with the cylinder turbulent wake analysis of Parezanovic and Cadot (2012) who also observed an increase of the global frequency when the control cylinder approaches the inner part of the shear layer. Same arguments can probably be used: the separated shear may reattach on the control sphere which concentrates the vorticity compared to that of the natural case. This thinner shear will lead to a higher frequency of the global mode. For larger values of the radial position $\left(r_{c}^{*}>0.45\right.$ in Fig. $\left.5 \mathrm{~d}\right)$, the control sphere must widen the wake which tends to decrease the frequency.

\section{References}

Berger, E., Scholz, D., Schumm, M., 1990. Coherent vortex structures in thewake of a sphere and a circular disk at rest and under forced vibrations. Journal of Fluids and Structures 4 (3), 231-257.

Cannon, S., Champagne, F., Glezer, A., 1993. Observations of large-scale structures in wakes behind axisymmetrical bodies. Experiments in Fluids 14 (6), 447-450.

Grandemange, M., Parezanović, V., Gohlke, M., Cadot, O., 2012. On experimental sensitivity analysis of the turbulent wake from an axisymmetric blunt trailing edge. Physics of fluids 24, 035106. 
Hill, D., 1992. A theoretical approach for analyzing the restabilization of wakes. NASA Technical Report No. 103858.

Howe, M., Lauchle, G., Wang, J., 2001. Aerodynamic lift and drag fluctuations of a sphere. Journal of Fluid Mechanics 436, 41-57.

Jang, Y. I. I., Lee, S. J., 2008. Piv analysis of near-wake behind a sphere at a subcritical reynolds number. Experiments in Fluids 44 (6), 905-914.

Luchini, P., Giannetti, F., Pralits, J., 2009. Structural sensitivity of the finiteamplitude vortex shedding behind a circular cylinder. IUTAM Symposium on Unsteady Separated Flows and their Control, 151-160.

Marquet, O., Sipp, D., Jacquin, L., 2008. Sensitivity analysis and passive control of cylinder flow. Journal of Fluid Mechanics 615, 221-252.

Meliga, P., Chomaz, J., Sipp, D., 2009a. Global mode interaction and pattern selection in the wake of a disk: a weakly nonlinear expansion. Journal of Fluid Mechanics 633, 159-189.

Meliga, P., Chomaz, J., Sipp, D., 2009b. Unsteadiness in the wake of disks and spheres: Instability, receptivity and control using direct and adjoint global stability analyses. Journal of Fluids and Structures 25 (4), 601-616.

Meliga, P., Pujals, G., Serre, E., 2012. Sensitivity of 2-d turbulent flow past a d-shaped cylinder using global stability. Physics of Fluids 24, 061701.

Mittal, R., Wilson, J., Najjar, F., 2002. Symmetry properties of the transitional sphere wake. AIAA journal 40 (3), 579-582.

Pao, H., Kao, T., 1977. Vortex structure in the wake of a sphere. Physics of Fluids 20, 187.

Parezanović, V., Cadot, O., 2012. Experimental sensitivity analysis of the global properties of a 2D turbulent wake. Journal of Fluid Mechanics 693, $115-149$.

Sakamoto, H., Haniu, H., 1990. A study on vortex shedding from spheres in a uniform flow. ASME, Transactions, Journal of Fluids Engineering 112, 386-392. 
Sakamoto, H., Haniu, H., 1995. The formation mechanism and shedding frequency vortices from a sphere in uniform shear-flow. Journal of Fluid Mechanics 287, 151-171.

Schouveiler, L., Brydon, A., Leweke, T., Thompson, M., 2004. Interactions of the wakes of two spheres placed side by side. European Journal of Mechanics-B/Fluids 23, 137-145.

Strykowski, P., Sreenivasan, K., 1990. On the formation and suppression of vortex sheddingat low Reynolds numbers. Journal of Fluid Mechanics 218, 71-107.

Szaltys, P., Chrust, M., Przadka, A., Goujon-Durand, S., Tuckerman, L. S., Wesfreid, J. E., 2012. Nonlinear evolution of instabilities behind spheres and disks. Journal of Fluids and Structures 28, 483-487.

Taneda, S., 1978. Visual observations of the flow past a sphere at Reynolds numbers between 104 and 106. Journal of Fluid Mechanics 85 (01), 187192.

Yoon, D.-H., Yang, K.-S., 2007. Flow-induced forces on two nearby spheres. Physics of Fluids 19, 098103.

Yun, G., Kim, D., Choi, H., 2006. Vortical structures behind a sphere at subcritical Reynolds numbers. Physics of Fluids 18, 015102. 